University of Nebraska - Lincoln

DigitalCommons@University of Nebraska - Lincoln

December 1987

\title{
Electrooptic and piezoelectric measurements in photorefractive barium titanate and strontium barium niobate
}

\author{
Stephen Ducharme \\ University of Nebraska - Lincoln, sducharme1@unl.edu \\ Jack Feinberg \\ University of Southern California - Los Angeles \\ R.R. Neurgaonkar \\ Rockwell International Science Center, Thousand Oaks, California
}

Follow this and additional works at: https://digitalcommons.unl.edu/physicsducharme

Part of the Physics Commons

Ducharme, Stephen; Feinberg, Jack; and Neurgaonkar , R.R., "Electrooptic and piezoelectric measurements in photorefractive barium titanate and strontium barium niobate" (1987). Stephen Ducharme Publications. 26.

https://digitalcommons.unl.edu/physicsducharme/26

This Article is brought to you for free and open access by the Research Papers in Physics and Astronomy at DigitalCommons@University of Nebraska - Lincoln. It has been accepted for inclusion in Stephen Ducharme Publications by an authorized administrator of DigitalCommons@University of Nebraska - Lincoln. 


\title{
Electrooptic and Piezoelectric Measurements in Photorefractive Barium Titanate and Strontium Barium Niobate
}

\author{
STEPHEN DUCHARME, JACK FEINBERG, MEMBER, IEEE, AND RATNAKER R. NEURGAONKAR
}

\begin{abstract}
We have measured the low-frequency ("unclamped") electrooptic and piezoelectric coefficients in undoped $\mathrm{BaTiO}_{3}$ and

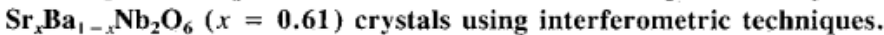
The contribution of the piezoelectric effect to the Pockels measurements is discussed. For an applied ac electric field in the range 0.1-200 $\mathrm{V} / \mathrm{cm}$, the electrooptic and piezoelectric effects are linear in the magnitude of the applied field and independent of its frequency in the range $10 \mathrm{~Hz}-100 \mathrm{kHz}$. The unclamped electrooptic coefficients of poled $\mathrm{BaTiO}_{3}$ single crystals are $r_{13}=19.5 \pm 1 \mathrm{pm} / \mathrm{V}$ and $r_{33}=97 \pm 7$ $\mathrm{pm} / \mathrm{V}$, and for strontium barium niobate are $r_{13}=\mathbf{4 7} \pm 5 \mathrm{pm} / \mathrm{V}$ and $r_{33}=235 \pm 21 \mathrm{pm} / \mathrm{V}$, all measured at a wavelength of $514.5 \mathrm{~nm}$ and at $T=23^{\circ} \mathrm{C}$. For the barium titanate samples the measured Pockels coefficient $r_{c} \equiv r_{33}-\left(n_{1} / n_{3}\right)^{3} r_{13}=79 \pm 6 \mathrm{pm} / \mathrm{V}$ is in good agreement with the value $r_{c}=76 \pm 7 \mathrm{pm} / \mathrm{V}$ computed from the above values of $r_{13}$ and $r_{33}$, where $n_{1}$ and $n_{3}$ are the ordinary and extraordinary indexes of refraction, respectively. The measured piezoelectric coefficient is $d_{23}$ $=+28.7 \pm 2 \mathrm{pm} / \mathrm{V}$ for barium titanate, and is $d_{23}=+24.6 \pm 2 \mathrm{pm} / \mathrm{V}$ for strontium barium niobate. We also measured the photorefractive coupling of two optical beams in the crystals, and we show that the dependence of the coupling strength on beam polarization is in fair agreement with the measured values of the Pockels coefficients.
\end{abstract}

\section{INTRODUCTION}

$\mathrm{P}$ HOTOREFRACTIVE crystals $\left(\mathrm{BaTiO}_{3}\right)$ and strontium

of barium

titanate $\left(\mathrm{Sr}_{x} \mathrm{Ba}_{1-x} \mathrm{Nb}_{2} \mathrm{O}_{6}\right)$ have proven useful for a variety of nonlinear optical applications [1] due, in part, to their large linear electrooptic (Pockels) coefficients. Recent models of the photorefractive effect show that the nonlinear coupling strength between optical beams is proportional to the Pockels coefficients and to the difference in the electron and hole photoconductivities of the crystal [2]-[6]. Consequently, accurate values of the Pockels coefficients are needed in order to determine the relative contribution

Manuscript received May 11, 1987; revised August 31, 1987. Work performed at the University of Southern California was supported in part by the Air Force Office of Scientific Research under Contract F49620-850110, and by the Joint Services Electronics Program under Contract F49620-85-C-0071. Work performed at Rockwell International was supported by DARPA under Contract N00014-82-C-2466.

S. Ducharme was with the Department of Physics, University of Southern California, University Park, Los Angeles, CA 90089. He is now with the Department of Physics, University of Utah, Salt Lake City, UT 84112.

J. Feinberg is with the Department of Physics, University of Southern California, University Park, Los Angeles, CA 90089.

R. R. Neurgaonkar is with the Rockwell International Science Center, Thousand Oaks, CA 91360.

IEEE Log Number 8717500. of electrons and holes to the photorefractive effect in these crystals.

Previously reported measurements of the electrooptic coefficients in $\mathrm{BaTiO}_{3}$ were made on flux-grown (Remieka Method) crystals [7]-[10] and not on the higher quality melt-grown (Czochralski Method) crystals now available [11]. The flux-grown crystals have relatively large concentrations of impurities, particularly iron, so that their dielectric and electrooptic properties may differ from those of the much purer melt-grown crystals. Additionally, previous interferometric measurements of the electrooptic coefficient $r_{13}$ of $\mathrm{BaTiO}_{3}$ were in error as they neglected the piezoelectric contribution to the phase change of a beam passing through the electrooptic crystal [12].

Here we present corrected interferometric measurements [13] of selected Pockels and piezoelectric coeffi-

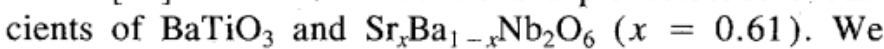
consider and eliminate the following possible sources of systematic error in the measured electrooptic coefficients: 1) the interplay between piezoelectric and Pockels effects, 2) space charge and electrode effects in the electrooptic measurements, 3) misalignment of the optical and the lowfrequency electric fields to the crystal axes (especially important in $\mathrm{BaTiO}_{3}$ because of its large coefficient $r_{42}$ coefficient), and 4) possible incomplete poling of the crystals.

The crystals were grown from a top-seeded melt (Czochralski method), cut, polished, and mechanically poled (to eliminate $90^{\circ}$ domains) [14]. We electrically poled the crystals (to eliminate $180^{\circ}$ domains) using a technique previously developed in poling numerous crystals [2]. The dimensions of the various crystals are given in Table I. The crystal faces were at right angles to within $\pm 1^{\circ}$ and cut normal to the principal axes of the crystal, as determined optically. The $c$ axis (spontaneous polarization axis) was at right angles to the face to within $\pm 1^{\circ}$. The $\mathrm{BaTiO}_{3}$ crystal faces were slightly convex and produced a mildly astigmatic lensing with focal lengths of 2 to $10 \mathrm{~m}$ along the $x$ axis. The crystals were without visible flaw and had a faint, olive-brown color.

$\mathrm{BaTiO}_{3}$ is tetragonal with $4 \mathrm{~mm}$ symmetry between $\sim 9$ and $\sim 127^{\circ} \mathrm{C}$. Letting the spatial coordinates $x-y-z$ be numbered 1-2-3, with the $c$ axis along the $z$ (or 3 ) direction, the nonzero electrooptic coefficients are (in the con- 
TABLE I

CRystal Dimensions (In Centimeters)

\begin{tabular}{llll}
\hline \hline crystal & $\mathbf{1}$ & $\mathbf{2}$ & 3(c-axis) \\
\hline BaTiO $_{3}$ & & & \\
SWISS & 0.420 & 0.480 & 0.455 \\
CAT & 0.435 & 0.560 & 0.540 \\
DOYLE & 0.235 & 0.565 & 0.445 \\
FREE & 0.540 & 0.530 & 0.730 \\
ROCKY & 0.420 & 0.420 & 0.370 \\
SBN:60 & 0.550 & 0.535 & 0.700 \\
LiNbO $_{3}$ & 0.445 & 0.500 & 0.510
\end{tabular}

(All dimensions are $\pm 0.005 \mathrm{~cm}$ )

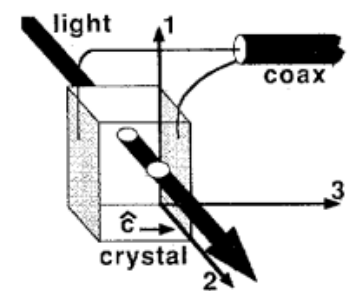

Fig. 1. Crystal geometry and coordinate system used in the electrooptic measurements. The electric field is applied along the 3 direction, the direction of the spontaneous polarization of the crystal ( $c$ axis). The optical beam propagates along the 2 direction.

tracted notation of [15] and [16]) $r_{13}=r_{23}, r_{33}$, and $r_{42}=$ $r_{51}$, and the corresponding piezoelectric coefficients are $d_{13}=d_{23}, d_{33}$, and $d_{42}=d_{51}$. (Here the positive 3 direction is defined as the direction of spontaneous polarization, which is in the direction of the applied poling electric field.) The crystal is birefringent with refractive indexes $n_{3}=2.431$ and $n_{1}=n_{2}=2.494$ at room temperature and at a vacuum wavelength $\lambda=514.5 \mathrm{~nm}$ [17], [18]. In all of our experiments the applied electric field and the light-induced space-charge field were along the $c$ axis. Optical beams were propagated along the $y$ direction and polarized along the $x$ direction (ordinary rays) or in the $z$ direction (extraordinary rays), as shown in Fig. 1.

The Pockels coefficients can be determined from the magnitude of the measured phase change as follows. An electric field $E$ applied across the crystal will alter the index ellipsoid by the electrooptic effect [15], [16]. The change in the refractive index $\Delta n_{i}$ for a beam polarized along the $i$ th crystal axis is

$$
\Delta n_{i}=-(1 / 2) n_{i}^{3} \sum_{j=1}^{3} r_{i j} E_{j}
$$

Note, however, that the same applied field can, by the piezoelectric effect [15], cause a change $\Delta L_{k}$ in the length $L_{k}$ of the crystal along the $k$ direction:

$$
\Delta L_{k}=\sum_{j=1}^{3} d_{k j} E_{j} L_{k}
$$

An electric field $E_{3}$ applied along the crystal's 3 direction will cause a change $\Delta \phi_{i}$ in the optical phase $\phi_{i}=$ $2 \pi n_{i} L_{2} / \lambda$ of a beam propagating along the 2 direction and polarized along the $i$ th axis:

$$
\begin{aligned}
\Delta \phi_{i} & =(2 \pi / \lambda) \Delta\left(n_{i} L_{2}\right)=(2 \pi / \lambda)\left(\Delta n_{i} L_{2}+n_{i} \Delta L_{2}\right) \\
& =\left(2 \pi L_{2} / \lambda\right)\left(-n_{i}^{3} r_{i 3} / 2+\left[n_{i}-1\right] d_{23}\right) E_{3}
\end{aligned}
$$

where $L_{2}$ is the length of the crystal along the 2 direction (the beam propagation direction), $i=1$ for ordinary rays and $i=3$ for extraordinary rays, and $\lambda$ is the vacuum wavelength. The negative sign on the electrooptic term in (3) comes from the definition of the electrooptic coefficients [15], [16].

The Pockels coefficients are determined by applying an electric field $E_{3}$ along the crystal's $c$ axis (the 3 axis) and measuring the resulting phase change impressed on an optical beam passing through the crystal. An argon-ion laser operating in a single longitudinal $\mathrm{TEM}_{00}$ mode supplied the optical beams to the homemade Mach-Zehnder interferometer shown in Fig. 2 [19]-[20]. A $1 \mathrm{~m}$ focal length lens was placed in the reference arm to approximate the lensing produced by the crystal, which was placed in the signal arm. The resulting interference pattern was a series of concentric ellipses. The optical intensities in the two arms of the interferometer were adjusted to be approximately equal at the output of the interferometer. The polarization of the optical beams could be made parallel or perpendicular to the $c$ axis of the crystal.

A dc voltage $\left(E_{3}^{\mathrm{dc}}=0-100 \mathrm{~V} / \mathrm{cm}\right)$ plus a small sinusoidal ac voltage ( $E_{3}^{\text {ac }}=0.1-200 \mathrm{~V} / \mathrm{cm}$ peak-to-peak $)$ at frequencies ranging from $10^{1}$ to $10^{5} \mathrm{~Hz}$ were applied to the silver paint electrodes on the two $c$ axis faces of the crystal. The applied voltage $E_{3}=E_{3}^{\mathrm{dc}}+E_{3}^{\mathrm{ac}}$ altered the phase of the beam passing through the crystal. This phase change was monitored at the output of the interferometer by a pinhole and detector, followed by a lock-in amplifier with an integrating time of $10 \mathrm{~s}$ and set to the same frequency as the applied ac electric field. The frequency response and linearity of the function generator, cables, detector, and lock-in amplifier were all carefully checked. A Babinet-Soleil compensator in one interferometer arm was adjusted so that with no ac voltage $\left(E_{3}^{\mathrm{ac}}=0\right)$ the output intensity at the center of the interference pattern was $I_{1}+I_{2}$, where $I_{1}$ and $I_{2}$ are the separate output intensities from each of the interferometer arms. This set the phase difference between the two interferometer arms to be $\pi / 2$ (modulo $2 \pi$ ), which was the most sensitive operating point of the interferometer. This bias point was measured at the start and at end of each $10 \mathrm{~s}$ experiment to insure that it had not drifted during data collection.

Turning on the applied ac electric field $\left(E_{3}^{\text {ac }} \neq 0\right)$ induced a small phase change $\Delta \phi_{i}$ in one arm of the interferometer, which caused an intensity change $\Delta I$ at the output of the interferometer given by

$$
\Delta I=-2\left(I_{1} I_{2}\right)^{1 / 2} \sin \left(\Delta \phi_{i}\right) \approx-2\left(I_{1} I_{2}\right)^{1 / 2} \Delta \phi_{i}
$$

for $\Delta \phi_{i}<<1$.

For a given applied field $E_{3}$, the ratio $\Delta I /\left(I_{1}+I_{2}\right)$ was measured to determine the phase change $\Delta \phi_{i}$ from (4). Note that the applied electric field $E_{3}$ in (3) causes the phase change $\Delta \phi_{i}$ via two distinct mechanisms: 1) the 


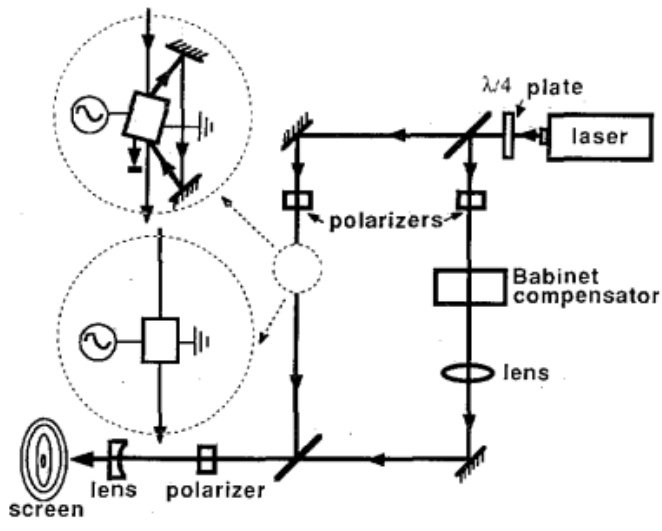

Fig. 2. Electrooptic interferometers. The Mach-Zehnder interferometer used to measure the electrooptic coefficients $r_{13}$ and $r_{33}$ (lower inset) and the piezoelectric coefficient $d_{13}$ (upper inset). The crystal is placed in one arm and a Babinet-Soleil compensator in the other arm. The crystal $c$ axis and the compensator slow axis are aligned in the plane of the figure. The polarization of the optical beams in the two polarizer arms are made the same by the separate polarizers. The output polarizer was aligned parallel to the input polarizers.

change in the crystal's refractive index (electrooptic effect), and 2) the change in the crystal's length along the path of the optical beam (piezoelectric effect). This is not to be confused with the difference between the unclamped and clamped Pockels coefficients, for which the crystal is either allowed (unclampled) or not allowed (clamped) to expand along the direction of the applied electric field. Here our experiments are performed with an ac field whose frequency is far below any acoustic resonance of the crystal, so our measurement yields a value for the unclamped Pockels coefficient. A high-frequency experiment would yield a clamped measurement, and in that case there would be no piezoelectric contribution to the measured phase change $\Delta \phi_{i}$.

In order to use (3) to determine the Pockels coefficients $r_{i j}$ from the measured phase change $\Delta \phi_{i}$, the piezoelectric length change $\Delta L_{2}$ must be measured independently. The piezoelectric coefficient $d_{13}=d_{23}$ was measured using the same interferometer shown in Fig. 2. However, instead of passing one beam through the crystal, the beam was reflected off of two opposite crystal faces, as shown in the upper inset of Fig. 2. This allowed a direct measurement of any change in the length of the crystal along the $2 \mathrm{di}$ rection due to the applied electric field along the 3 direction. By reflecting the light off both crystal faces, the sensitivity to any translation of the crystal was eliminated, and the sensitivity to the crystal's length change was doubled [13]. The sign and the magnitude of the measured piezoelectric coefficient $d_{23}$ was then used to compute the Pockels coefficients $r_{i 3}$ using (1)-(4). Table II shows the results of the low-frequency electrooptic and piezoelectric measurements in the various crystals. Our value of $d_{23}=$ $+28.7 \pm 2 \mathrm{pm} / \mathrm{V}$ for the piezoelectric coefficient of $\mathrm{BaTiO}_{3}$ has the same magnitude but opposite sign of measurements using piezoelectric resonances on similar crystals [22], [23]. We find that in both barium titanate and strontium barium niobate, the application of an electric
TABLE II

Electrooptic Measurements in Photorefractive Crystals at 514.5 nm (ExCEPT as Noted), AN AC Electrical MODUlation of $1000 \mathrm{~Hz}$, and a Crystal Temperature $T=23^{\circ} \mathrm{C}$. The Indicated ERrors are Standard Deviations as Determined by Repeated MEasurements

\begin{tabular}{|c|c|c|c|c|c|c|c|}
\hline Crystal & $\begin{array}{l}r_{13} \\
\text { (picomet } \\
\text { meas }\end{array}$ & $\begin{array}{l}r_{33} \\
\text { erivolit) }\end{array}$ & $\mid \begin{array}{r}r_{c}=r_{33}- \\
\text { (picon } \\
\text { measure }\end{array}$ & $\begin{array}{l}\left(n_{1} / n_{3}\right)^{3} r_{13} \\
\text { eter Nolt) } \\
\text { d computed }\end{array}$ & $\begin{array}{l}\frac{n_{3}{ }^{3} r_{33}}{n_{1}{ }^{3} r_{13}} \\
\text { computed }\end{array}$ & $\begin{array}{l}=g_{3} / g_{1} \\
\text { measured }\end{array}$ & $\mathbf{d}_{13}$ \\
\hline $\begin{array}{l}\mathrm{BATIO}_{3} \\
\text { SWISS } \\
\text { CAT } \\
\text { DOYLE } \\
\text { FREE } \\
\text { ROCKY }\end{array}$ & $\begin{array}{c}19.4 \pm 1 \\
19.7 \pm 1 \\
19.4 \pm 1\end{array}$ & \begin{tabular}{l}
$97 \pm 7$ \\
$99 \pm 5$ \\
\hdashline$-\overline{94.4 \pm 8}$
\end{tabular} & \begin{tabular}{c}
$79 \pm 6$ \\
\hdashline$-\cdots$ \\
\hdashline$\cdots$ \\
\hdashline$-\cdot-$
\end{tabular} & \begin{tabular}{c}
$76 \pm 8$ \\
$78 \pm 6$ \\
\hdashline$-:$ \\
$74 \pm 9$
\end{tabular} & \begin{tabular}{l}
$4.7 \pm .5$ \\
$4.7 \pm .4$ \\
\hdashline..- \\
$4.6 \pm .5$
\end{tabular} & $\begin{array}{l}3.6 \pm .2 \\
3.3 \pm .2 \\
3.5 \pm .2 \\
3.4 \pm .5 \\
2.8 \pm .5\end{array}$ & \begin{tabular}{l}
$28.7 \pm 3$ \\
$28.7 \pm 1$ \\
\hdashline$-\cdots$ \\
\hdashline$-\cdots$
\end{tabular} \\
\hline SBN:60 & $46.7 \pm 5$ & $235 \pm 20$ & $\cdots$ & $\ldots$ & $5.0 \pm .8$ & $5.8 \pm 1$ & $24.6 \pm 1.5$ \\
\hline$\underset{*}{\mathrm{LiNbO}_{3}}$ & $\begin{array}{l}10.1 \pm 3 \\
9.620\end{array}$ & $\begin{array}{l}28.8 \pm 1 \\
30.920\end{array}$ & -....... & $\frac{17.7 \pm 1.5}{---}$ & $\begin{array}{l}2.9 \pm .3 \\
2.9\end{array}$ & $\begin{array}{l}2.1 \pm .3 \\
3.127\end{array}$ & $\begin{array}{l}\left|d_{13}\right|<1 \\
-1^{28,29}\end{array}$ \\
\hline
\end{tabular}

- Values in the last row are taken at $\lambda=632.8 \mathrm{~nm}$ from Refs. $20,27-29$

field in the 3 direction caused the crystal to expand in the 2 direction, when the electric field was applied in the same direction as the original electric field used to pole the crystal. From (2) and the above definition for the positive direction of the electric field, this implies a positive value for the piezoelectric coefficient $d_{23}$.

A number of tests were performed on two of the $\mathrm{BaTiO}_{3}$ crystals (CAT and SWISS) to determine the role, if any, of surface layers, space-charge fields, and photorefractive effects on the interferometrically measured values of the electrooptic coefficients. Space charge limited conduction occurs for frequencies below the dielectric relaxation rate, which was $<10 \mathrm{~Hz}$ in our $\mathrm{BaTiO}_{3}$ samples at the optical intensities used here $\left(<<1 \mathrm{~W} / \mathrm{cm}^{2}\right.$.) We checked for space-charge effects on the measured electrooptic coefficients $r_{13}$ and $r_{33}$ in several ways: 1) the frequency of the applied ac field was varied from $10 \mathrm{~Hz}$ to $100 \mathrm{kHz}$ with no measurable change in the amplitude of the detected signal; 2) the magnitude of the applied ac field was varied from 0.1 to $200 \mathrm{~V} / \mathrm{cm}$, as shown in Fig. 3, and the magnitude of the applied dc field varied from 0 to $100 \mathrm{~V} / \mathrm{cm}$ with no measurable change in the computed coefficients; 3 ) the signal was independent of the position and size of the optical beam in the crystal; 4) the signal was independent of the intensity of the interferometer beam and of the intensity of another beam (incoherent with the first beam) that uniformly illuminated the crystal; and 5) the signal was independent of the presence of a photorefractive grating created in the crystal by two "writing" beams coherent with each other but incoherent with the interferometer beam. Any space-charge effects would have shown up in tests 1)-4) above. Test 5) eliminated any possible electrostrictive effects caused by the large $(\sim 1000 \mathrm{~V} / \mathrm{cm})$ spatially-periodic photorefractive electric field induced by a photorefractive grating.

The possibility of piezoelectric resonances contributing to our measurements was also ruled out. The frequencies of the acoustic normal modes of a crystal of length $L$ are $f_{m}=m v / 2 L$ (ignoring damping) where $v$ is the velocity of sound in the crystal, and $m$ is an integer. The speed of 


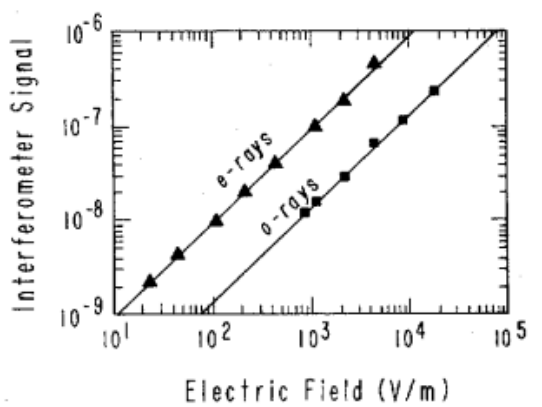

Fig. 3. Log-log plot of the interferometer signal versus the amplitude of the ac electric field applied across the $c$ axis of the "SWISS" $\mathrm{BaTiO}_{3}$ crystal, using the interferometer of Fig. 2. The Pockels coefficients $r_{13}$ and $r_{33}$ are determined from the $y$ intercepts of the plots. The straight lines have a slope of unity.

sound in $\mathrm{BaTiO}_{3}$ at room temperature ranges [22]-[24] from 3000 to $6500 \mathrm{~m} / \mathrm{s}$ (depending on the particular acoustic mode) so that $f_{1} \geq 300 \mathrm{kHz}$ in all of the crystals studied, which is much larger than the frequencies of our ac fields. As an experimental check, we modulated the ac voltage on the crystal at frequencies up to $100 \mathrm{kHz}$ and saw no change in either the electrooptic or piezoelectric response, indicating that we were indeed far from any piezoelectric resonances.

To confirm our measured values of the Pockels coefficients, we also measured the change in the crystal birefringence induced by an applied electric field, and so determined the combination of electrooptic coefficients $r_{c} \equiv$ $r_{33}-\left(n_{1} / n_{3}\right)^{3} r_{13}$. An argon-ion laser beam $(\lambda=514.5$ $\mathrm{nm}$ ) initially polarized at an angle of $45^{\circ}$ with respect to both the 1 and 3 axes of the crystal was propagated along the 2 axis of the crystal, as shown in Fig. 4. An electric field $E_{3}=E_{3}^{\mathrm{dc}}+E_{3}^{\mathrm{ac}}$ was applied along the 3 axis of the crystal. The linear birefringence of the crystal $\left(n_{1}-n_{3}\right.$ $\approx 0.06$ ) plus the birefringence induced by the applied electric field caused a phase difference $\Delta \phi$ between the 1 and 3 components of the transmitted optical field. A Babinet-Soleil compensator following the crystal was adjusted so that $\Delta \phi=\pi / 2(\operatorname{modulo} 2 \pi)$ when $E_{3}^{\text {ac }}=0$, in which case the intensity transmitted through the second polarizer was half of its maximum value. With the ac component $E_{3}^{\text {ac }}$ now made nonzero, the intensity of the beam transmitted through the analyzing polarizer is given by (4) with

$$
\Delta \phi=(2 \pi L / \lambda)\left[d_{23}\left(n_{3}-n_{1}\right)-n_{3}^{3} r_{c} / 2\right] E_{3}^{\text {ac }} .
$$

Because the birefringence of the crystal is small, the first term in (5) is negligible compared to the second, and measuring the intensity of the slope of the curve in Fig. 5 gives a value for the combined Pockels coefficient $r_{c}=$ $79 \pm 6 \mathrm{pm} / \mathrm{V}$. This is in good agreement with the value of $r_{c} \equiv r_{33}-\left(n_{1} / n_{3}\right)^{3} r_{13}=76 \pm 7 \mathrm{pm} / \mathrm{V}$ calculated from our individually measured Pockels coefficients, as listed in Table II.

The ratio $n_{3}^{3} r_{33} / n_{1}^{3} r_{13}$ of the Pockels coefficients was also measured separately using two photorefractive techniques [1], [25]. First, the magnitude of photorefractive

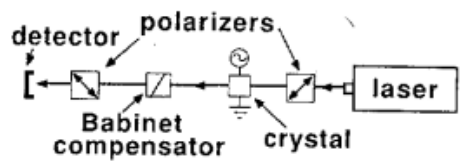

Fig. 4. Measurement on the combined Pockels coefficient $r_{c}$. The polarization of the optical beam at the entrance face of the crystal is at an angle of $45^{\circ}$ to the crystal's $c$ axis. The Babinet-Soleil compensator, with its slow axis parallel to the crystal $c$ axis, is used to adjust the polarization of the beam incident on the crossed output analyzer.

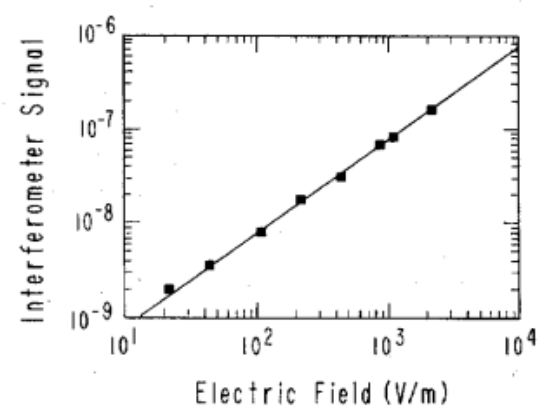

Fig. 5. Log-log plot of the transmitted signal versus the amplitude of the ac electric field applied across the $c$ axis of the "SWISS" $\mathrm{BaTiO}_{3}$ crystal, using the setup of Fig. 4. The combined Pockels coefficient $r_{c}$ is determined from the $y$ intercept of the plot. The straight line has a slope of unity.

two-beam coupling gain $g$ was measured in each $\mathrm{BaTiO}_{3}$ crystal for two extraordinary polarized beams $\left(g=g_{3}\right)$ or two ordinary polarized beams $\left(g=g_{1}\right)$, where the gain $g$ is defined as the intensity increase of the transmitted probe beam due to the presence of a strong pump beam. The experiment was performed at $514.5 \mathrm{~nm}$ using beam diameters of $\sim 3 \mathrm{~mm}$, an external beam-crossing angle of a few degrees, the $k$-vector of the photorefractive grating aligned along the $c$ axis of the crystal, and with the intensity of the pump beam (incident intensity $=10^{-1} \mathrm{~W} / \mathrm{cm}^{2}$ ) much larger than the intensity of the probe beam (incident intensity $=10^{-3} \mathrm{~W} / \mathrm{cm}^{2}$ ). Only the peak value of the transmitted probe beam power was recorded, as the signal fluctuated in time due to vibration of the optical components. Note that this measurement is sensitive to errors caused by pump beam depletion, beam nonuniformity, a photovoltaic field in the crystal, or crystal misalignment. The ratio of the two-beam coupling strengths $g_{3} / g_{1}$ for the two polarization is given in Table II. According to theory [25] $g_{3} / g_{1}=n_{3}^{3} r_{33} / n_{1}^{3} r_{13}$. Note, however, that our measured ratio of the two-beam coupling gains $g_{3} / g_{1}$ is significantly less than the ratio $n_{3}^{3} r_{33} / n_{1}^{3} r_{13}$ computed from our interferometrically measured Pockels coefficients $r_{33}$ and $r_{13}$. One explanation is that beam-fanning [26] of the extraordinary polarized beams is reducing the coupling gain $g_{3}$. Another (more interesting) explanation is that there are different excitation cross sections for electrons and for holes in these $\mathrm{BaTiO}_{3}$ samples, and that the cross sections for the two species are polarization dependent, as discussed below.

The ratio $n_{3}^{3} r_{33} / n_{1}^{3} r_{13}$ of the Pockels coefficients was also measured by another technique, in which the diffraction efficiency of a photorefractive grating is measured 
simultaneously for ordinary and extraordinary beams. A weak probe beam (incident intensity $=10^{-3} \mathrm{~W} / \mathrm{cm}^{2}$ ) polarized at $45^{\circ}$ to the $y z(2-3)$ plane was made to intersect a strong pump beam (incident intensity $=10^{-1} \mathrm{~W} / \mathrm{cm}^{2}$ ) polarized either as an ordinary ray along the 1 direction, or as an extraordinary ray in the 2-3 plane, in a $\mathrm{BaTiO}_{3}$ crystal. The two beams wrote a photorefractive grating. The strong pump beam was then blocked, and the weak probe beam (which was so weak that it negligibly erased the grating) was Bragg-deflected off of the grating. The diffraction efficiency for each of the polarizations was measured by placing a polarization analyzer on the deflected beam. By polarizing the probe beam at $45^{\circ}$, the diffraction efficiency of either the ordinary or the extraordinary beam could be measured simultaneously from the same refractive-index grating without changing the alignment of any beams. (Both the ordinary and extraordinary polarization components of the probe beam are automatically Bragg-matched to the grating, independent of the polarization of the pump beam). The ratio of the 2-3 and the 1 components of the diffracted beam should be $\left(n_{3}^{3} r_{33} / n_{1}^{3} r_{13}\right)^{2}$. Note that this ratio is insensitive to the optical beam intensities or to the optical alignment. A measurement in the "DOYLE" crystal of $\mathrm{BaTiO}_{3}$ gave $n_{3}^{3} r_{33} / n_{1}^{3} r_{13}=3.6 \pm 1$ for an $o$-polarized pump and 5.9 \pm 2 for an $e$-polarized pump. (This should be compared to the measured value $g_{3} / g_{1}=3.5 \pm 0.2$ for the ratio of two-beam coupling gains in the same crystal.) The dependence on writing-beam polarization can be explained if there is significant hole-electron competition in these samples, and if the cross section for optical excitation of these charges differs for the two optical polarizations. If true, then the conductivities of the various species could be determined by a series of such experiments. This will be pursued in a separate work.

As a final check on our experimental techniques, we measured the known electrooptic coefficients of a $\mathrm{LiNbO}_{3}$ crystal, and found good agreement with the values reported in the literature, as shown in Table II.

Our revised values for the Pockels coefficients of barium titanate and strontium barium niobate can now be used in current models for charge transport [5]-[6] to accurately determine the relative contributions of electron and hole transport to the photorefractive effect in these crystals. For example, in [25] it was thought that holeelectron competition was absent in $\mathrm{BaTiO}_{3}$ because the measured two-beam coupling gain agreed with the theoretical gain calculated from the Pockels coefficient $r_{13}=$ 8 (which was the best value available at the time) and assuming only one species of charge carriers (holes). However, our new value $r_{13}=19.5$ implies that, had there really been no hole-electron competition in those experiments, the measured coupling gain should have been about 2.5 times larger. Therefore those measurements actually proved that electrons and holes had comparable photoconductivities in that $\mathrm{BaTiO}_{3}$ crystal. Indeed, our revised values for $r_{13}$ and $r_{33}$ of $\mathrm{BaTiO}_{3}$ imply that electrons and holes have comparable photoconductivities in all of the $\mathrm{BaTiO}_{3}$ crystals studied in [2] and [3], in spite of the oxidation or reduction treatments used in those experiments to eliminate such competition.

\section{ACKNOWLEDGMENT}

We thank R. W. Hellwarth, A. Marrakchi, K. R. MacDonald, and A. R. Tanguay, Jr. for useful discussions.

\section{REFERENCES}

[1] See for example, Optical Phase Conjugation, R. A. Fisher, Ed. New York, Academic, 1983, ch. 11, and references therein.

[2] S. Ducharme and J. Feinberg, J. Opt. Soc. Amer. B, vol. 3, p. 283, 1986.

[3] M. B. Klein and R. N. Schwartz, J. Opt. Soc. Amer. B., vol. 3, p. 293,1986 .

[4] R. Orlowski and E. Kratzig, Solid State Commun., vol. 27, p. 1351, 1978.

[5] F. P. Strohkendl, J. M. C. Jonathan, and R. W. Hellwarth, Opt. Lett., vol. 11, p. 312, 1986.

[6] G. C. Valley, J. Appl. Phys., vol. 59, p. 3363, 1986.

[7] I. P. Kaminov, Appl. Phys. Lett, vol. 7, p. 123, 1965, vol. 8, p. 54, 1966 Erratum; I. P. Kaminov, Appl. Phys. Lett., vol. 8, p. 305, 1966.

[8] A. R. Johnston and J. M. Weingart, J. Opt. Soc. Amer., vol. 55, p. $828,1965$.

[9] A. R. Johnston, Appl. Phys. Lett., vol. 7, p. 195, 1965.

[10] - , J. Appl. Phys., vol. 12, p. 3501, 1971.

[11] G. Godefroy, P. Lompre, and C. Dumas, Mater. Res. Bull., vol. 12, p. 165,1977

[12] S. Ducharme, J. Feinberg, and R. R. Neurgaonkar, J. Opt. Soc. Amer. $A$, vol. 2; p. P108, 1985.

[13] The results published in the 1986 OSA Annual Meeting summary (S Ducharme, J. Feinberg, and R. R. Neurgaonkar, J. Opt. Soc. Amer. $A$, vol. 3, p. P25, Dec. 1986) were incorrect due to a neglected factor of 2 in determining the piezoelectric coefficients.

[14] Sanders Assoc., Nashua, $\mathrm{NH}$ ( $\mathrm{BaTiO}_{3}$ crystals).

[15] P. Nye, Physical Properties of Crystals. London, England: Univ. Oxford Press, 1967.

[16] A. Yariv, Quantum Electronics, 2nd Edition. New York: Wiley, 1975.

[17] I. Camlibel, M. Didomenico, and S. H. Wemple, J. Phys. Chem. Solids, vol. 31, p. 1417, 1970.

[18] S. H. Wemple, M. Didomenico, and I. Camlibel, J. Phys. Chem. Solids, vol. 29, p. 1797, 1968

[19] K. Onuki, N. Uchida, and T. Saku, J. Opt. Soc. Amer., vol. 62, p. 1030,1972 .

[20] K. Takizawa and M. Okada, J. Opt. Soc. Amer., vol. 72, p. 809, 1982.

[21] - J. Opt. Soc. Amer. B, vol. 2, p. 289, 1985.

[22] M. A. Schaefer, H. Schmitt, and A. Dorr, Ferroelect., vol. 69, p. 253,1986

[23] D. Berlincourt and H. Jaffe, Phys. Rev., vol. 111, p. 143, 1958.

[24] G. Shirane, J. D. Axe, and J. Harada, Phys. Rev. B, vol. 2, p. 3651, 1970.

[25] J. Feinberg, D. Heiman, A. R. Tanguay, Jr., and R. W. Hellwarth, J. Appl. Phys., vol. 51, p. 1297, 1980; -, J. Appl. Phys., vol. 52, p. 537,1981 , Erratum.

[26] J. Feinberg, J. Opt. Soc. Amer., vol. 72, p. 46, 1982.

[27] S. I. Stepanov, A. A. Kamshilin, and M. P. Petrov, Soc. Phys. Solid State, vol. 19, p. 418, 1977.

[28] T. Yamada, N. Niizeki, and H. Toyoda, Japan J. Appl. Phys., vol. 6 , p. $151,1967$.

[29] A. W. Warner and A. A. Ballman, Proc. IEEE, vol. 55, p. 450, 1967. 


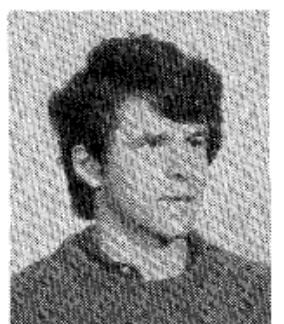

Stephen Ducharme was born in Greenfield, MA, in 1959. He received the B.S. degree in physics from the University of Lowell, Lowell, MA, in 1981 and the M.A. and Ph.D. degrees in physics from the University of Southern California, Los Angeles, in 1982 and 1986, respectively.

$\mathrm{He}$ is presently a postdoctoral assistant at the University of Utah, Salt Lake City, where he has studied the optical properties of chalcogenide glasses and amorphous silicon. $\mathrm{He}$ is now investigating the high frequency magnetic susceptibil-

ity of the high-temperature superconductor $\mathrm{Y}_{1} \mathrm{Ba}_{2} \mathrm{Cu}_{3} \mathrm{O}_{7}$.

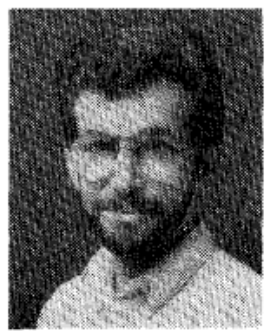

Jack Feinberg was born and raised in New York, NY. He received the A.B. degree in Physics with Honors from Columbia University, New York, in 1972, and the Ph.D. degree from the University of California, Berkeley, in 1977, where he worked on molecular spectroscopy.

He joined the University of Southern California, Los Angeles, as a postdoctoral researcher, joined their Physics faculty in 1981, and became an Associate Professor in 1985. His interests include anything optical, especially optical phase conjugation, nonlinear optics, spectroscopy, applications of optics to medicine, and making movies.

Prof. Feinberg is a member of various professional and veterinary societies.

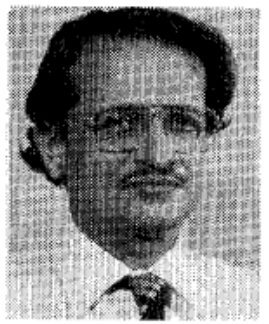

Ratnakar R. Neurgaonkar received the B.S. (with honors), M.S., and Ph.D. degrees in solidstate chemistry from Poona University, Poona, India, in 1962, 1963, and 1967, respectively.

Currently, he is manager of the Ferroelectric Materials Department Rockwell International Science Center, Thousand Oaks, CA. At Rockwell, he has been directing the ferroelectric materials research and development program for various device applications, including electrooptic, photorefractive and pyroelectric imagers, SAW's, multimeter-wave, and piezoelectric transducer applications. He and $\mathrm{W}$. Cory have developed various growth techniques for ferroelectric crystals/ films, and recently they successfully demonstrated the growth of opticalquality doped and undoped $\mathrm{Sr}_{1}-{ }_{x} \mathrm{Ba}_{x} \mathrm{Nb}_{2} \mathrm{O}_{6}$ and $\mathrm{BSKNN}$ single crystals using the Czochralski technique. Besides ferroelectric materials, he has also been interested in magnetics, luminescence, and laser crystal development work. He is the author or coauthor of more than 70 research publications.

Dr. Neurgaonkar is a member of the American Ceramic Society, the Electrochemical Society, the American Association for Crystal Growth, and other various professional societies. 